\title{
Exclude Body Weight Gain Data From
} Statistics Indicator

National Cancer Institute

\section{Source}

National Cancer Institute. Exclude Body Weight Gain Data From Statistics Indicator. NCI

Thesaurus. Code C119766.

Specifies whether the body weight gain result values should be excluded from the statistical analysis. 